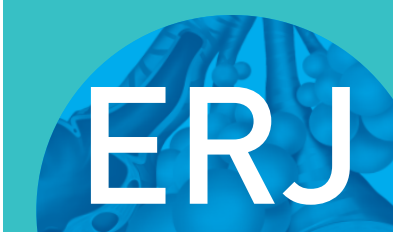

open research
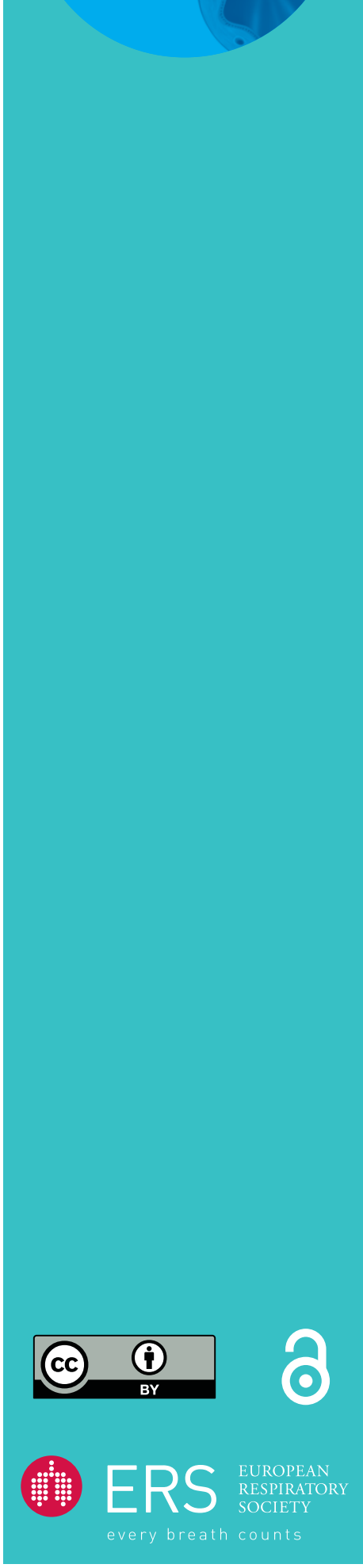

\section{The Living with Pulmonary Fibrosis questionnaire in progressive fibrosing interstitial lung disease}

\author{
Jeffrey Swigris (101, Katelyn Cutts ${ }^{2}$, Natalia Male ${ }^{3}$, Michael Baldwin $^{3}$, \\ Klaus B. Rohr ${ }^{3}$ and Donald M. Bushnell (1) ${ }^{2}$
}

Affiliations: ${ }^{1}$ National Jewish Health, Denver, CO, USA. ${ }^{2}$ Evidera, Bethesda, MD, USA. ${ }^{3}$ Boehringer Ingelheim International $\mathrm{GmbH}$, Ingelheim am Rhein, Germany.

Correspondence: Jeffrey Swigris, National Jewish Health, 1400 Jackson Street, Denver, Colorado, USA. E-mail: SwigrisJaNJHealth.org

ABSTRACT The Living with Idiopathic Pulmonary Fibrosis (L-IPF) questionnaire was developed with substantial input from patients with idiopathic pulmonary fibrosis (IPF) to assess symptoms and healthrelated quality of life (HRQoL). Because IPF is the prototypical chronic fibrosing interstitial lung disease (ILD) with a progressive phenotype, we expanded applicability of the L-IPF by deleting the word "idiopathic", creating the L-PF (Living with Pulmonary Fibrosis) questionnaire, and then assessed its relevance among patients with progressive fibrosing ILDs in one-to-one interviews.

Patients in the USA and Germany with any progressive fibrosing ILD other than IPF were asked about their disease and symptoms, completed the 44-item L-PF questionnaire (comprising two modules that assess symptoms and impacts of disease) and then answered a series of debriefing questions. Interviews were recorded, transcribed and coded for qualitative content analysis.

20 patients were interviewed, but time constraints meant not all were asked about all items. The most frequent diagnoses were rheumatoid arthritis-associated ILD $(25 \%)$ and mixed connective tissue diseaseassociated ILD (20\%). Almost all patients endorsed the symptoms assessed by the L-PF: shortness of breath (19 out of 20 patients), cough (19 out of 20) and fatigue (18 out of 20). Most patients endorsed impacts of progressive fibrosing ILD on activities of daily living, physical well-being, sleep, emotional well-being, and social aspects of their lives. Most patients had an overall positive impression of the Symptoms module and understood items as intended. All seven patients asked understood the items of the Impacts module.

The L-PF contains concepts relevant and important to patients with progressive fibrosing ILD, and items are understood as intended.

@ERSpublications

The Living with Pulmonary Fibrosis questionnaire contains relevant and important concepts that are understood by patients with all forms of progressive fibrosing interstitial lung disease https://bit.ly/3eb7M7Q

Cite this article as: Swigris J, Cutts $\mathrm{K}$, Male $\mathrm{N}$, et al. The Living with Pulmonary Fibrosis questionnaire in progressive fibrosing interstitial lung disease. ERJ Open Res 2021; 7: 00145-2020 [https://doi.org/10.1183/23120541.00145-2020].

This article has supplementary material available from openres.ersjournals.com

Received: 25 March 2020 | Accepted: 25 Feb 2021

Copyright $\odot$ The authors 2021. This version is distributed under the terms of the Creative Commons Attribution Licence 4.0. 


\section{Introduction}

Interstitial lung diseases (ILDs) are a large group of diffuse, parenchymal lung disorders. A proportion of patients with chronic fibrosing ILD develop a progressive phenotype associated with increasing fibrosis on chest imaging, greater symptom burden, deteriorating lung function, declining physical functional capacity, worsening health-related quality of life (HRQoL) and often early death [1,2]. Idiopathic pulmonary fibrosis (IPF) is the archetypal progressive fibrosing ILD [3]. Patients with any form of ILD, including those caused by environmental or occupational exposures, systemic autoimmune diseases or idiopathic interstitial pneumonias, may develop a progressive fibrosing phenotype [2]. Progressive fibrosing ILDs other than IPF have pathogenetic mechanisms and disease behaviour similar to IPF, including progressive symptoms, effects on HRQoL, risk for early mortality, and response to antifibrotic drugs [2, 4]. Given the commonalities among the progressive fibrosing ILDs (IPF and others), it is useful to study them as a group rather than by individual aetiologies [5].

The most common and burdensome symptoms of progressive fibrosing ILD, dyspnoea, cough and fatigue, are strong drivers of HRQoL impairment $[6,7]$. Patient-reported outcome measures (PROMs) are useful for assessing how a condition or its treatment affects the way patients feel and function in their daily lives [7]. PROMs are also important for assessing certain effects of therapies. Thus, PROMs yield patient-centred information that other metrics of disease severity (e.g. pulmonary physiology, imaging) cannot. Various PROMs that assess symptoms and health status have been validated in patients with IPF, including the Cough and Sputum Assessment Questionnaire (CASA-Q) [8], the UCSD Shortness of Breath Questionnaire (SOBQ) [8], the original St. George's Respiratory Questionnaire (SGRQ) [9] and its IPF-specific version (the SGRQ-I) [10], and the King's Brief Interstitial Lung Disease Questionnaire (K-BILD) [11]. However, none of these, nor any other PROM, was developed specifically for assessing patients with progressive fibrosing ILD; the K-BILD was developed to measure health status in patients with a wide range of inflammatory or fibrosing ILDs, including IPF.

The Living with IPF (L-IPF) questionnaire was developed to assess symptoms and HRQoL specifically in patients with IPF [12] and incorporated direct input from patients with IPF at each stage of development. Some of the qualitative data used to develop the A Tool to Assess Quality of life in IPF (ATAQ-IPF) [13] informed the development of the L-IPF, together with additional data and analyses conducted for the L-IPF [14]. Because IPF is a subset of progressive fibrosing ILD, and because all forms of progressive fibrosing ILD have similar symptoms, natural history and risk of death, we expect the L-IPF to be equally relevant to patients with any form of progressive fibrosing ILD. To improve inclusivity and broaden its target population, the L-IPF was very slightly modified to create the Living with Pulmonary Fibrosis (L-PF) questionnaire; it is identical to the L-IPF, except that the two mentions of the word "idiopathic" in the L-IPF were removed. The L-PF has been used to assess symptoms and HRQoL in a multicentre clinical trial of nintedanib in patients with progressive fibrosing ILD (INBUILD), and analyses of the L-PF data are underway [5].

The aim of this study was to interview patients with progressive fibrosing ILDs other than IPF, to gather qualitative data on their perceptions of how the condition affects their lives, to debrief after completion of the L-PF, and, ultimately, to assess the relevance of the L-PF and its fitness for the purpose of evaluating the symptoms and impacts of progressive fibrosing ILD.

\section{Methods}

\section{Study design}

This was a qualitative study involving one-on-one interviews with adult patients with progressive fibrosing ILD from two clinic sites in the USA and one in Germany. Clinical site staff identified potential patients and invited them in person or via telephone. All patients provided written informed consent. Prior to initiation, the study protocol received institutional review board approval in the USA and Germany (US Advarra Study Number: Pro00023847; Approval: December 19, 2017; Germany EC Study Number: 8053_BO_K_2018; Approval: August 7, 2018).

\section{Patients}

Patients were aged $\geqslant 18$ years with a confirmed diagnosis of progressive fibrosing ILD. They were required to have fibrosis in $>10 \%$ of the lung on high-resolution computed tomography (HRCT), diffusing capacity of the lungs for carbon monoxide $\left(D_{\mathrm{LCO}}\right)$ corrected for haemoglobin $(\mathrm{Hb}) \geqslant 30 \%$ and $<80 \%$ of predicted normal, and forced vital capacity (FVC) $\geqslant 45 \%$ predicted. Patients also needed to fulfil one of the following criteria for progressive fibrosing ILD within 24 months of screening despite previous ILD treatment: clinically significant decline in FVC $\%$ predicted based on a relative decline of $\geqslant 10 \%$; marginal decline in FVC \% predicted based on a relative decline of $5-<10 \%$ combined with worsening of respiratory symptoms or increasing extent of fibrotic changes on chest imaging; or worsening respiratory symptoms as 
well as increasing extent of fibrotic changes on HRCT. Patients also had to be willing and able to attend an interview either in person or via telephone.

Patients were excluded if they had pre-existing medical conditions that might confound reporting (in the opinion of the investigator; e.g. acute coronary syndrome), had a clinical diagnosis of IPF based on the 2011 guidelines of the American Thoracic Society, European Respiratory Society, Japanese Respiratory Society, and the Latin American Thoracic Association, were currently taking nintedanib or pirfenidone, had a history of alcohol or drug abuse (unless in full remission for $>6$ months prior to interview), had confirmed cognitive impairment or were otherwise judged to be unable to participate by the study principal investigator(s).

\section{Interviews}

Interviews were conducted at clinical sites or, if it was not possible to do so in person, via telephone. Three interviewers experienced in qualitative interview methodology led the discussion using a standardised, semi-structured guide (see Appendix) that was developed in collaboration with clinical experts and translated into German.

Firstly, in the concept elicitation phase of the interview, patients were asked about their disease and whether they had any symptoms that day in order to help assess whether the symptoms included in the L-PF were commonly reported in this group. If patients did not spontaneously mention shortness of breath, cough or fatigue, they were asked about each symptom specifically. Patients were then asked a series of questions about the frequency and severity of each symptom. Following the open-ended discussion, patients were asked to complete the paper version of the L-PF questionnaire and then answer a series of questions about the L-PF to assess their understanding and interpretation of items, thoughts about the recall period, and gather feedback on the relevance of its content. Interviewees were also asked to complete the K-BILD and answer questions about it, but in this article, we focus only on the L-PF. All interview sessions were audio recorded and transcribed using a professional transcription service. Transcripts were reviewed for content and participant-identifying information was removed.

The entire process took approximately $90 \mathrm{~min}$, including consent, interview and sociodemographic questionnaire. If interviews began to run out of time, the L-PF Symptoms measure was prioritised.

\section{L-PF questionnaire}

The questionnaire consists of 44 items divided into two modules: Symptoms (23 items) and Impacts (21 items). The Symptoms module assesses shortness of breath, cough and fatigue in the past $24 \mathrm{~h}$. For each of the shortness of breath items, respondents are asked to rate shortness of breath resulting from doing activities of varying intensities that span a range of metabolic demands. Because some respondents may not perform certain activities, each item first asks whether the respondent performed the activity in the last $24 \mathrm{~h}$; if they did, they proceed to rating their shortness of breath; if they did not perform the activity in the last $24 \mathrm{~h}$, they are asked whether they did not have the opportunity to do it (e.g. a respondent who does not have stairs in their home may not have had the opportunity to climb a flight of stairs in the last $24 \mathrm{~h}$ ) or whether they avoided the activity because it was too difficult to perform. The Impacts module assesses multiple aspects of HRQoL with a recall period of 1 week. Items in both modules have response options on a five-option numeric rating score with an anchor of 0 "Not at all" to 4 "Extremely". Overall scores range from 0 to 100 , with higher numbers indicating a greater impairment. The L-PF questionnaire was translated into German by the MAPI Research Trust using a rigorous translation process $[15,16]$ and patients in Germany completed a German version of the questionnaire.

\section{Analysis}

The interviews were coded for qualitative content analysis using ATLAS.ti software (version 8.0) and using methodology described by WILLIS [17]. A coding dictionary of key concepts was developed, and investigators tested the concepts by coding several transcripts and comparing between investigators. The dictionary was refined until the coding was consistent. One coder then completed the coding of all transcripts using the constant comparative method, an iterative coding approach moving between consecutive transcripts and any new codes that emerge [18].

Quotations from interviews are included in this article to illustrate patient responses.

\section{Results}

\section{Patients}

In total, 20 patients were included; 15 in the USA and five in Germany. All interviews were conducted in person apart from four in the USA that were conducted by telephone. The mean age was 70.1 years (median 72.5; range $48-81$ ); $50 \%$ of patients were female, and $85 \%$ of patients were white (table 1 ). Mean 


\section{TABLE 1 Patient demographics and disease characteristics}

\section{Characteristic}

\begin{tabular}{|c|c|}
\hline Patients $\mathbf{n}$ & 20 \\
\hline \multicolumn{2}{|l|}{ Age years } \\
\hline Mean \pm SD & $70.1 \pm 9.5$ \\
\hline Median (range) & $72.5(48-81)$ \\
\hline Female $\mathrm{n}(\%)$ & $10(50)$ \\
\hline \multicolumn{2}{|l|}{ Smoking status $\mathrm{n}(\%)$} \\
\hline Current smoker & $1(5)$ \\
\hline Previous smoker & 7 (35) \\
\hline Non-smoker & $12(60)$ \\
\hline \multicolumn{2}{|l|}{ Duration since ILD diagnosis based on imaging years } \\
\hline Mean \pm sD & $4.3 \pm 3.1$ \\
\hline Median (range) & $3.0(1.8-12.5)$ \\
\hline \multicolumn{2}{|l|}{ Underlying ILD diagnosis n (\%) } \\
\hline Rheumatoid arthritis-associated ILD & $5(25)$ \\
\hline Mixed connective tissue disease & $4(20)$ \\
\hline Hypersensitivity pneumonitis & $3(15)$ \\
\hline Systemic sclerosis-associated ILD & $2(10)$ \\
\hline Respiratory bronchiolitis with ILD & $2(10)$ \\
\hline Other fibrotic ILD" & $4(20)$ \\
\hline \multicolumn{2}{|l|}{ Diagnosed with usual interstitial pneumonia pattern ${ }^{\natural} \mathrm{n}(\%)$} \\
\hline Yes & $6(30)$ \\
\hline No & $14(70)$ \\
\hline \multicolumn{2}{|l|}{ FVC $\%$ predicted } \\
\hline Mean \pm SD & $67.0 \pm 13.6$ \\
\hline Median (range) & $66.5(47-95)$ \\
\hline \multicolumn{2}{|l|}{$D_{\text {Lco }} \%$ predicted } \\
\hline Mean \pm SD & $51.6 \pm 9.7$ \\
\hline Median (range) & $53.0(35-66)$ \\
\hline \multicolumn{2}{|l|}{ Comorbidities $\mathrm{n}(\%)^{+}$} \\
\hline History of unstable or deteriorating cardiac disease & $1(5)$ \\
\hline COPD & $4(20)$ \\
\hline Sleep disorders & $3(15)$ \\
\hline GORD & $12(60)$ \\
\hline Articular joint involvement & $1(5)$ \\
\hline Other & $15(75)$ \\
\hline None & $2(10)$ \\
\hline Supplemental oxygen use $\mathrm{n}(\%)$ & 7 (35) \\
\hline \multicolumn{2}{|l|}{ BMI } \\
\hline Mean \pm SD & $26.8 \pm 4.6$ \\
\hline Median (range) & $26.9(20-36)$ \\
\hline \multicolumn{2}{|c|}{$\begin{array}{l}\text { ILD: interstitial lung disease; FVC: forced vital capacity; } D_{\text {LCo: }} \text { diffusing capacity of the lungs for carbon } \\
\text { monoxide; GORD: gastro-oesophageal reflux disease; BMI: body mass index. \#: systemic lupus (n=1), } \\
\text { byssinosis ( } n=1) \text {, drug toxicity ( } n=1) \text {, inflammatory bowel disease-related ILD }(n=1) ;{ }^{\text {: }} \text { : defined based on the } \\
\text { American Thoracic Society/European Respiratory Society/Japanese Respiratory Society/Latin American } \\
\text { Thoracic Association } 2011 \text { quidelines: }{ }^{+} \text {: not mutually exclusive. }\end{array}$} \\
\hline
\end{tabular}

time since ILD diagnosis based on imaging was 4.3 years (median 3.0; range 1.8-12.5). Median FVC \% predicted was $66.5 \%$ (range $47-95$ ) and median $D_{\text {LCO }} \%$ predicted was $53 \%$ (range 35-66). The most frequent diagnoses were rheumatoid arthritis-associated ILD (25\% of patients), mixed connective tissue disease-associated ILD (20\%), and hypersensitivity pneumonitis (15\%) (table 1).

\section{Concept elicitation}

Almost all patients spontaneously reported shortness of breath (19 out of 20 patients), with one endorsing it when probed. When asked about the frequency of shortness of breath, three patients said it was constant, three described it as occurring a few times a day, two as occurring almost every day/when active, and four patients when exerting themselves. Four patients described their shortness of breath as severe and not good, one as between moderate and severe, two as not bad/not very severe, and one as mild to medium (see quotation in table 2). 


TABLE 2 Illustrative quotations from the interviews
Popic
Patient quotation
Shortness of breath
Interviewer: How would you describe the severity of the difficulty
breathing?
Patient: It's not good; it stops me in my tracks. Like I said I have to sit, I
have to stop what I'm doing and recover. So it's definitely changed
every aspect of my life.
Interviewer: We'll talk about the cough first. How would you describe
that cough?
Patient: It is most like a dry hacky cough, I don't cough up anything and
it's very tiring. I can cough sometimes, like I said, I cough, some days
I cough a lot and I have like coughing spells where I cough for it
seems forever, but I'm sure it's probably just a minute or so at a
time. Like I said, it's just something that's really more like an
aggravation and tiring, it tires you out.
Interviewer: Okay. You sleep several times a day, but how often
would you say you just feel tired, in general?
Patient: I would say all the time.
Interviewer: Every day?
Patient: Other than when l'm sleeping, I'm just tired.
Patient: You can tell that these questions were written by people who
really know what it is like ... I have to say. Really well done.
Patient: I was thinking about the last 24 h, because I, as a sick person,
you always only think day to day, only on the last 24 h. The last 24 h, I
have calculated exactly what I did yesterday at this time. So, it
worked wonderfully. Fits perfectly.
Patient: I'd have to say they were all relevant, yes, I had some where,
no, I did not do, but I would say they would all be relevant.
breath items

Cough was reported by the majority of patients (19 out of 20 patients) either spontaneously (nine) or when probed (10) (table 3). Nine patients described their cough as occurring daily. When asked about the severity of their cough, one participant described it as severe, one as affecting activities, three as not severe but nagging/annoying, and two as "usually not bad". See example quotation in table 2.

Fatigue was reported by the majority of patients (18 out of 20 patients) either spontaneously (nine) or when probed (nine). Three patients described it as being constant (table 2), three as occurring at least once a day, and three as occurring when pushing or exerting themselves or doing activities.

Other symptoms mentioned included heavy breathing, joint pain, weight loss, loss of appetite, chest rattling and heaviness (two participants each), trouble swallowing (one participant), body aches and feeling a heavy weight on their body (one participant), and light-headedness, nail clubbing, pain, weakness, chest pressure, numbness, headaches, hoarseness, disorientation and chest pain (one patient each).

Patients were also asked how having progressive fibrosing ILD affected their day-to-day life. Most patients endorsed various impacts related to their physical functioning, including activities of daily living, sleep, emotional well-being and social aspects of their lives. Of the 18 patients who reported effects on daily living; the majority (16 out of $18 ; 89 \%$ ) had difficulty with completing housework, yard work or daily chores. 16 patients (out of $20 ; 80 \%$ ) reported impairments in physical functioning, most frequently difficulty in walking (five out of 16;31\%) and exercising (four out of 16;25\%). Eight patients (out of 20; $40 \%$ ) reported negative impacts of progressive fibrosing ILD on sleep, and 14 out of 20 (70\%) reported

TABLE 3 Number of patients reporting shortness of breath, cough and fatigue in the concept elicitation phase of the interview

\begin{tabular}{lccc} 
Symptom & Brought up spontaneously $\mathbf{n}$ & Endorsed when probed $\mathbf{n}$ & Total $(\mathbf{N}=\mathbf{2 0})$ \\
\hline Shortness of breath & 19 & 1 & 20 \\
Cough & 9 & 10 & 19 \\
Fatigue & 9 & 9 & 18
\end{tabular}


negative effects on their emotional health, including stress and anxiety (four out of 14; 29\%), getting frustrated easily and feelings of concern/worry and fear (two out of 14 for each; 14\%).

\section{Cognitive debriefing of the L-PF}

Due to respondent burden and time constraints, not all patients debriefed on both modules, so items were not discussed individually. The majority of patients (13 out of 15) had an overall positive impression of the Symptoms module of the L-PF (table 2). One patient reported both positive and negative impressions, and one did not directly answer the question.

Of the 15 patients who were asked about the time period they were thinking of while completing the Symptoms module, six reported they were thinking of the previous $24 \mathrm{~h}$ (as intended in the questionnaire) (table 2), two reported the "previous $24 \mathrm{~h}$ and 7 days depending on the question". Other time periods mentioned by one patient each were: all the time; the last 2 days; now; morning until now; $2-4$ weeks; last few days; and the last 14 days.

Of the patients asked, the majority demonstrated understanding of the items when asked to explain what they meant (cough: 15 out of 16 patients; shortness of breath: 16 out of 17; fatigue: 14 out of 15).

Eighteen patients were asked about the relevancy of the shortness of breath items; 10 patients (56\%) reported that all the items were relevant to them, whereas eight patients (44\%) said certain items were relevant to their experience (table 2). Most of these patients named one item that they felt was not relevant, and two patients stated that most were not relevant (figure 1). One patient responded that they were unsure whether the items were relevant to them.

Seven patients were asked about the meaning of items from the Impacts module. All seven interpreted the items associated with shortness of breath, cough and fatigue as the developer intended by stating that they were asking about how symptoms impacted their daily life and activities. All seven also interpreted the quality of life items as intended; they described quality of life as being able to do what you wanted throughout the day, including socialising and running errands. The five patients who were asked about the response options for the quality of life items understood the numeric response options and were able to provide detailed explanations for each response on the differing scales. Seven patients were asked about the Global items. They stated that the items were asking about physical health and quality of life as well as the general state of well-being. All seven demonstrated an understanding of the response options for these items.

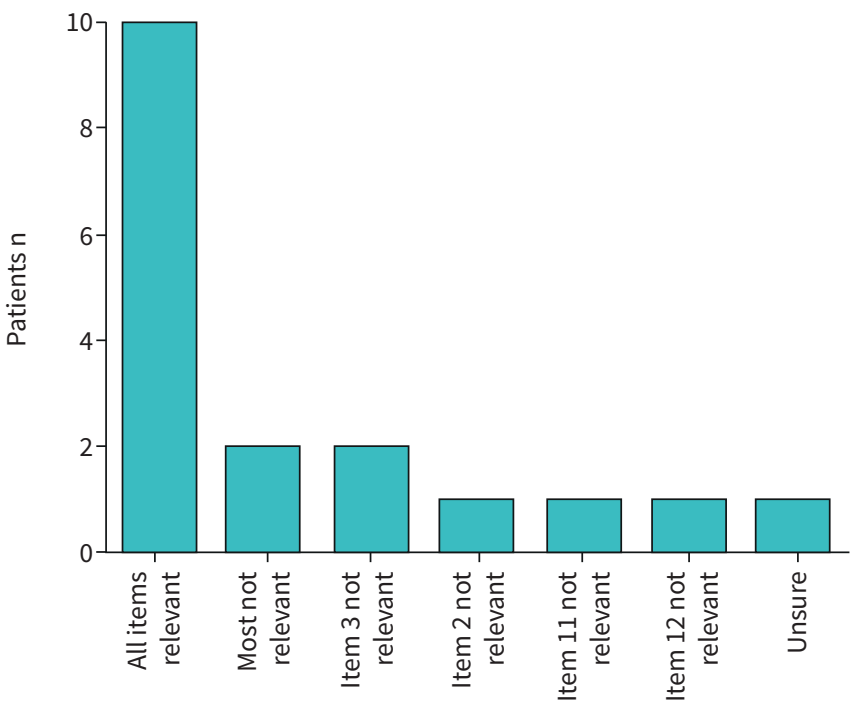

FIGURE 1 Relevancy of the shortness of breath items of the living with pulmonary fibrosis (L-PF) questionnaire. Shortness of breath items in the L-PF questionnaire were: Item 2: "Did you walk up one flight of stairs in the last 24 h?" Yes, "How short of breath did walking up one flight of stairs make you?"; Item 3 : "Over the last $24 \mathrm{~h}$, how short of breath have you been while sitting down, relaxing, reading, or watching TV?"; Item 11: "Over the last $24 \mathrm{~h}$, how short of breath were you after eating a meal or snacks?"; Item 12: "Did you lift and carry a light load (e.g., less than 10 lbs) a short distance (e.g., from one room to another) in the last $24 \mathrm{~h}$ ?" Yes, "How short of breath did lifting and carrying a light load a short distance make you?". L-PF: living with pulmonary fibrosis. 


\section{Discussion}

Overall, these data show that the L-PF contains relevant and important concepts and is understood by patients with various forms of progressive fibrosing ILD. The three symptoms addressed by the L-PF, shortness of breath, cough and fatigue, were endorsed by almost all of the patients, supporting their relevance in patients with progressive fibrosing ILD. Other symptoms were mentioned by only a few interviewees, suggesting that they are not important to most patients in the target population, though a larger study would be needed to determine their prevalence. Patients generally interpreted all items in the Symptoms module as the developer intended.

Patients largely understood that the items in the Symptoms module were asking about their symptoms over the previous $24 \mathrm{~h}$, and there was positive feedback about the length of the recall period. Although symptoms may vary day to day, the rationale for the shorter-than-typical recall is that it likely decreases the bias of responses, and short recall periods are encouraged by regulatory agencies (e.g. the US Food and Drug Administration). This is one key difference between the L-PF and the K-BILD questionnaire, which has a 2-week recall [11].

Although a smaller number of patients were interviewed about the Impacts module, they all interpreted the items as intended and showed an understanding of the response options for the items.

The vast majority of clinical trials in ILDs have used FVC as the primary endpoint, either alone or as one facet of a composite endpoint. While FVC is regarded as an easily collected, valid and reproducible measure of physiological impairment, with very good performance characteristics, it does not capture (with any degree of granularity) many aspects of disease relevant to patients, such as how they feel and function in their daily lives [2]. Hence the addition of a validated PROM can provide extremely useful information alongside spirometry measurement.

The L-PF was included as a PROM in the INBUILD clinical trial, which tested the effects of nintedanib in patients with progressive fibrosing ILDs [5]. Analyses of L-PF data are currently underway. Patients in the current study met inclusion criteria for the INBUILD trial [5] and had similar demographic and clinical characteristics [4]. Our findings, that the concepts in the L-PF are relevant and important to patients with progressive fibrosing ILD, support the validity of the L-PF results in the INBUILD trial population and the use of this questionnaire to assess symptoms and HRQoL in patients with progressive fibrosing ILD in general. The identical content of the L-IPF and L-PF questionnaires, and the fact that IPF is a subcategory of progressive fibrosing ILD, would suggest that an IPF-specific questionnaire (e.g. the L-IPF) may not be needed, and moving forward, the L-PF could be used in trials, whether enrolment is limited only to patients with IPF or expanded to include patients with any form of progressive fibrosing ILD.

Although healthcare practitioners may not have time in regular visits to use lengthy patient questionnaires, the Symptoms module of the L-PF is relatively short (23 items) and captures the three most relevant and burdensome symptoms in patients with ILDs. Thus, if future validation work confirms acceptable performance in this setting, it could be suitable for use in clinical practice.

Our study has some limitations. Due to the nature of the open-ended discussions and time constraints of the interviews, not every patient responded to every item on the L-PF, so some data are missing; in particular, the cognitive debrief of the Impacts module was only completed in a small proportion of patients. It was important to ascertain what symptoms patients mentioned spontaneously before using the questionnaire; however, conducting the concept elicitation phase prior to the completion of the questionnaires may have affected patients' responses to the L-PF. It is also possible that completing both the L-PF and the K-BILD questionnaires in the same session may have confounded patients' answers about the time periods.

Despite these limitations, the results suggest that the L-PF is an innovative tool that taps relevant domains and includes items that are understood as the developer intended. Although additional testing and longitudinal validation is needed, we believe that the L-PF provides researchers interested in testing therapies for progressive fibrosing ILD with a disease-specific tool useful for assessing outcomes important to patients.

Acknowledgements: The authors meet criteria for authorship as recommended by the International Committee of Medical Journal Editors. The authors received no direct compensation related to the development of the manuscript. Writing, editorial support, and formatting assistance was provided by Claire Scofield of Meditech Media, which was contracted and funded by Boehringer Ingelheim International $\mathrm{GmbH}$ (BI). BI was given the opportunity to review the manuscript for medical and scientific accuracy as well as intellectual property considerations.

Conflict of interest: J. Swigris is a paid consultant for Lung Therapeutics, an unpaid consultant for Boehringer Ingelheim, and he receives grant support from Genentech and Boehringer Ingelheim. He is on the advisory board of Live Fully, Inc., which has a patent pending. K. Cutts reports grants from Boehringer Ingelheim during the conduct of 
the study. N. Male is an employee of Boehringer Ingelheim. M. Baldwin is an employee of Boehringer Ingelheim. K.B. Rohr is an employee of Boehringer Ingelheim. D.M. Bushnell reports grants from boehringer ingelheim during the conduct of the study.

Support statement: The study was funded by Boehringer Ingelheim International GmbH. Funding information for this article has been deposited with the Crossref Funder Registry.

\section{References}

1 Cottin V, Hirani NA, Hotchkin DL, et al. Presentation, diagnosis and clinical course of the spectrum of progressive-fibrosing interstitial lung diseases. Eur Respir Rev 2018; 27: 180076.

2 Cottin V, Wollin L, Fischer A, et al. Fibrosing interstitial lung diseases: knowns and unknowns. Eur Respir Rev 2019; 28: 180100.

3 Raghu G, Collard HR, Egan JJ, et al. ATS/ERS/JRS/ALAT Committee on Idiopathic Pulmonary Fibrosis. An official ATS/ERS/JRS/ALAT statement: idiopathic pulmonary fibrosis: evidence-based guidelines for diagnosis and management. Am J Respir Crit Care Med 2011; 183: 788-824.

4 Flaherty KR, Wells AU, Cottin V, et al. Nintedanib in progressive fibrosing interstitial lung diseases. $N$ Engl J Med 2019; 381: 1718-1727.

5 Flaherty KR, Brown KK, Wells AU, et al. Design of the PF-ILD trial: a double-blind, randomised, placebo-controlled phase III trial of nintedanib in patients with progressive fibrosing interstitial lung disease. BMJ Open Respir Res 2017; 4: e000212.

6 Fischer A, Distler J. Progressive fibrosing interstitial lung disease associated with systemic autoimmune diseases. Clin Rheumatol 2019; 38: 2673-2681.

7 Swigris JJ, Brown KK, Abdulqawi R, et al. Patients' perceptions and patient-reported outcomes in progressive-fibrosing interstitial lung diseases. Eur Respir Rev 2018; 27: 180075.

8 Gries KS, Esser D, Wiklund I. Content validity of CASA-Q cough domains and UCSD-SOBQ for use in patients with Idiopathic Pulmonary Fibrosis. Glob J Health Sci 2013; 5: 131-141.

9 Swigris JJ, Brown KK, Behr J, et al. The SF-36 and SGRQ: Validity and first look at minimum important differences in IPF. Respir Med 2010; 104: 296-304.

10 Prior TS, Hoyer N, Shaker SB, et al. Validation of the IPF-specific version of St. George's Respiratory Questionnaire. Respir Res 2019; 20: 199.

11 Patel AS, Siegert RJ, Brignall K, et al. The development and validation of the King's Brief Interstitial Lung Disease (K-BILD) health status questionnaire. Thorax 2012; 67: 804-810.

12 Graney B, Johnson N, Evans CJ, et al. Living with idiopathic pulmonary fibrosis (L-IPF): developing a patient-reported symptom and impact questionnaire to assess health-related quality of life in IPF. Am J Respir Crit Care Med 2017; 195: A5353.

13 Swigris JJ, Wilson SR, Green KE, et al. Development of the ATAQ-IPF: a tool to assess quality of life in IPF Health Qual Life Outcomes 2010; 8: 77.

14 Swigris JJ, Andrae DA, Churney T, et al. Development and initial validation analyses of the Living with Idiopathic Pulmonary Fibrosis (L-IPF) questionnaire. Am J Respir Crit Care Med 2020; 202: 1689-1697.

15 Acquadro C, Bayles A, Juniper E. Translating patient-reported outcome measures: a multi-step process is essential. J Bras Pneumol 2014; 40: 211-212.

16 Acquadro C, Conway K, Girourdet C, et al. Linguistic Validation Manual for Patient-Reported Outcomes (PRO) Instruments. Lyon, France, MAPI Research Trust, 2004.

17 Willis GB. Analysis of the Cognitive Interview in Questionnaire Design: Understanding Qualitative Research. New York, NY, Oxford University Press, 2015.

18 Boeije H. A purposeful approach to the constant comparative method in the analysis of qualitative interviews. Quality and Quantity 2002; 36: 391-409. 